\title{
Quantum noise and correlations in resonantly enhanced wave mixing based on atomic coherence
}

\author{
M. D. Lukin ${ }^{1,2,3}$, A. B. Matsko ${ }^{2,3}$, M. Fleischhauer ${ }^{2,4}$, and M. O. Scully ${ }^{2,3}$ \\ ${ }^{1}$ ITAMP, Harvard-Smithsonian Center for Astrophysics, Cambridge, MA 02138, \\ ${ }^{2}$ Department of Physics, Texas A $6 M$ University, College Station, Texas 77843-4242, \\ ${ }^{3}$ Max-Planck-Institut für Quantenoptik, Garching, D-85748, Germany, \\ ${ }^{4}$ Sektion Physik, Universität München, D-80333 München, Germany
}

We investigate the quantum properties of fields generated by resonantly enhanced wave mixing based on atomic coherence in Raman systems. We show that such a process can be used for generation of pairs of Stokes and anti-Stokes fields with nearly perfect quantum correlations, yielding almost complete (i.e. 100\%) squeezing without the use of a cavity. We discuss the extension of the wave mixing interactions into the domain of a few interacting light quanta.

One of the intriguing and potentially useful aspects of nonlinear optical phenomena is their ability to suppress intrinsic quantum fluctuations [1]. However, the efforts to exploit these properties were hindered, either by the small values of nonlinearities in available optical crystals, or by absorption losses and the associated noise in resonant atomic systems with large nonlinearities. For example, four-wave mixing is known to result, in principle, in squeezed-state generation or non-classical correlations [2], but all experimental realizations reported to date showed rather limited noise reduction and required the use of cavities [3].

The work of the past few years has shown that substantial improvements in resonant nonlinear optics can be achieved by utilizing the concepts of quantum coherence and interference [4.5]. The aim of the present contribution is to demonstrate the usefulness of this regime of nonlinear optical enhancement for applications involving quantum correlations and reduction of quantum noise. As an example, we consider here four-wave mixing in resonant Raman systems [5], where atomic phase coherence can be used to generate a large nonlinearity and at the same time suppress resonant absorption. Recent theoretical [6] and experimental work [7] demonstrated that the efficient nonlinear interactions in this system can lead to mirrorless parametric oscillation, where pairs of counter-propagating Stokes and anti-Stokes photons are generated spontaneously from noise. We here show that under certain, very realistic conditions this process can be considered as ideal from the viewpoint of quenching of quantum noise. As a result, the generated Stokes and anti-Stokes field components can possess practically perfect quantum correlations, leading e.g. to an almost complete suppression of the quantum fluctuations in one quadrature of a combined mode (i.e. 100\% squeezing). We point out that this can be achieved even in the case when the intensity of the driving fields approaches, under realistic experimental conditions, the few-photon level. These results, together with recent studies on strongly interacting photons [9], single-photon switching [10] and few photon quantum control [11], show that a truly new regime of nonlinear optics involving just a few interacting light quanta is feasible.

Physically, such a performance of the nonlinear media is due to the possibility of eliminating the resonant absorption and associated noise processes via atomic coherence. Furthermore, the associated large linear dispersion is very important for achieving phase matching [7] and plays a key role in the reduction of the oscillator linewidth [8] which will be discussed in detail elsewhere. The present results open new interesting possibilities for applications as diverse as novel frequency standards and gravity-wave detection on one hand and quantum-information processing on the other 12.

In the present paper we discuss the noise properties of electromagnetic waves propagating in a medium consisting of double- $\Lambda$ atoms as shown in Fig. 1 in a four-wave mixing configuration. Four optical waves are tuned to the vicinity of the corresponding optically allowed transitions. These fields include two counter-propagating driving fields with frequencies $\nu_{d 1}, \nu_{d 2}$ and Rabi-frequencies $\Omega_{1}$ and $\Omega_{2}$, and two probe fields (anti-Stokes and Stokes) with carrier frequencies $\nu_{1}=\nu_{d 1}+\omega_{0}$ and $\nu_{2}=\nu_{d 1}-\omega_{0}$, where $\omega_{0}=\omega_{b 1}-\omega_{b 2}$ is the ground-state frequency splitting. The probe fields are described quantum mechanically in an effective 1-D model. The fields interact via the long-lived coherence on the dipole-forbidden transition between the metastable ground states $\left|b_{1}\right\rangle$ and $\left|b_{2}\right\rangle$.

We here utilize a Langevin approach in which collective atomic variables and fields are described by timeand position- dependent stochastic differential equations with $\delta$ - correlated Langevin forces 13. . The present approach develops from the semiclassical analysis of Ref.[6], in which, in particular, a phase transition to mirrorless parametric oscillation was noted. We now proceed with an analysis of the quantum fluctuations in such a system. We obtain stochastic equations which, for undepleted pump fields, can easily be solved by Fouriertransformation. 
We introduce slowly-varying dimensionless field variables $\hat{E}_{1,2}(z, t)$ which contain only modes propagating in the $+z$ and $-z$ direction respectively

$$
\begin{aligned}
& \hat{E}_{1}(z, t)=\sum_{k>0} a_{k}(t) \mathrm{e}^{i\left(k-k_{1}\right) z} \mathrm{e}^{i \nu_{1} t}, \\
& \hat{E}_{2}(z, t)=\sum_{k>0} a_{-k}(t) \mathrm{e}^{-i\left(k-k_{2}\right) z} \mathrm{e}^{i \nu_{2} t},
\end{aligned}
$$

where $k_{1,2}=\nu_{1,2} / c$. Following the approach of [13] we derive stochastic differential equations for c-number analogues of the fields $\hat{E} \rightarrow E$ and collective atomic operators of the medium consisting of the four-state atoms shown in Fig.1. Apart from the stochastic noise sources these equations have a form identical to the semiclassical density matrix equations for such an atomic system. The diffusion coefficients for noise correlations are derived using the fluctuation-dissipation theorem and generalized Einstein relations. We find that the propagation of the Fourier-components of Stokes and anti-Stokes fields $E_{1}^{*}(z, \omega), E_{2}(z, \omega)$ is governed by the (c-number) equations

$$
\begin{aligned}
& \left(\frac{\partial}{\partial z}+\frac{i \omega}{c}\right) E_{1}^{*}(z, \omega)=-i \frac{k_{1}}{2 \epsilon_{0}} P_{1}^{*}(z, \omega), \\
& \left(-\frac{\partial}{\partial z}+\frac{i \omega}{c}\right) E_{2}(z, \omega)=i \frac{k_{2}}{2 \epsilon_{0}} P_{2}(z, \omega) .
\end{aligned}
$$

Here the $P_{l}$ 's are the c-number variables proportional to the corresponding polarizations in the appropriate units, and the Fourier-transform is defined as $F^{(*)}(\omega)=$ $1 / \sqrt{2 \pi} \int d t \mathrm{e}^{-i \omega t} F^{(*)}(t)$. Solving the equations of motion for the atomic variables in lowest order of the Stokes and anti-Stokes fields we find:

$$
\begin{aligned}
& P_{l}(z, \omega)=\epsilon_{0} \chi_{l l}(z, \omega) E_{l}(z, \omega) \\
& \quad+\epsilon_{0} \chi_{l m}(z, \omega) e^{i \Delta \vec{k} \cdot \vec{r}} E_{m}^{*}(z, \omega)-f_{l}(z, \omega) / k_{l},
\end{aligned}
$$

where $\{l, m\}=\{1,2\}$ and $m \neq l . \Delta \vec{k}$ is a possible geometrical phase mismatch, $\chi_{l l}(z, \omega)$ are the self-coupling and $\chi_{l m}(z, \omega)(m \neq l)$ the cross-coupling $\left(\chi^{(3)}\right.$-type $)$ susceptibilities of the medium [6]. Both do not depend on the amplitudes of Stokes and anti-Stokes fields but are functions of the drive-fields and thus in general spacedependent. $f_{1,2}(z, \omega)$ are noise sources, which are $\delta$ correlated in frequency and position. In the following we assume $\Delta \vec{k}=0$. Note, however, that a non-zero phase mismatch can easily be compensated in the present system by a small detuning of the Stokes and anti-Stokes fields from two-photon resonance. Thus the equations of motion for the Fourier-components of the fields at frequency $\omega$ are:

$$
\frac{d}{d z}\left[\begin{array}{c}
E_{1}^{*} \\
E_{2}
\end{array}\right]=i\left[\begin{array}{ll}
a_{11} & a_{12} \\
a_{21} & a_{22}
\end{array}\right]\left[\begin{array}{c}
E_{1}^{*} \\
E_{2}
\end{array}\right]+\frac{i}{2 \epsilon_{0}}\left[\begin{array}{l}
f_{1}^{*} \\
f_{2}
\end{array}\right],
$$

where $a_{1 j} \equiv a_{1 j}(z, \omega)=-k_{1} \chi_{1 j}^{*}(z, \omega) / 2-\delta_{j 1} \omega / c$, and $a_{2 j} \equiv a_{2 j}(z, \omega)=-k_{2} \chi_{2 j}(z, \omega) / 2+\delta_{j 2} \omega / c$.
In order to solve the inhomogeneous boundary-value problem we assume undepleted driving fields and transform away their remaining space dependence due to the refractive index. Thus $a_{i j}(z, \omega) \rightarrow a_{i j}(\omega)$. Assuming vacuum input $\left(E_{1}^{*}(0)=0, E_{2}(L)=0\right)$ at both sides of the medium of length $L$ we eventually find:

$$
\begin{aligned}
E_{1}^{*}(L) & =\int_{0}^{L} d z^{\prime} \frac{i f_{1}^{*}\left(z^{\prime}\right) M\left(z^{\prime}\right) \eta+a_{12} f_{2}\left(z^{\prime}\right) \sin \left(\eta z^{\prime}\right)}{2 \epsilon_{0} M(L) \eta e^{i \tilde{a}\left(z^{\prime}-L\right)}}, \\
E_{2}(0) & =\int_{0}^{L} d z^{\prime} \frac{a_{21} \tilde{f}_{1}^{*}\left(z^{\prime}\right) \sin \left(\eta z^{\prime}\right)-i \tilde{f}_{2}\left(z^{\prime}\right) M\left(z^{\prime}\right) \eta}{2 \epsilon_{0} M(L) \eta e^{i \tilde{a}\left(L-z^{\prime}\right)}},
\end{aligned}
$$

where we have dropped the frequency dependence. $\tilde{f}_{i}(z)=f_{i}(L-z), \tilde{a}=\left(a_{11}+a_{22}\right) / 2, a=\left(a_{22}-a_{11}\right) / 2$, $\eta=\sqrt{a^{2}+a_{12} a_{21}}$, and $M(z, \omega)=\cos (\eta z)+i a / \eta \sin (\eta z)$. These expressions predict infinite growth of the Stokes and anti-Stokes fields from vacuum when

$$
M(L, \omega) \rightarrow 0 \quad \text { or } \quad \tan (\eta L)=i \frac{\eta}{a},
$$

which is the oscillation condition 6, 14].

Let us proceed now with a special case in which one of the driving fields (say $\Omega_{1}$ ) is tuned near resonance with the corresponding single-photon transition $b_{2} \rightarrow a_{1}$, whereas the second driving field $\Omega_{2}$ has a detuning $\Delta \gg$ $\left|\Omega_{2}\right|$ from the transition $b_{1} \rightarrow a_{2}$. For simplicity assume also equal Rabi-frequencies of the driving fields $\left|\Omega_{1}\right|=$ $\left|\Omega_{2}\right|=|\Omega|$. In this case most of the population is in the lower state $b_{1}$, and there is almost no absorption of the driving fields. For small Fourier frequencies (close to the two-photon resonance) we find :

$$
a_{11}=\frac{\kappa\left[-\omega+i \gamma_{0}\right]}{|\Omega|^{2}}-\frac{\omega}{c}, a_{12}=a_{21}=\frac{\kappa}{\Delta}, a_{22}=\frac{\omega}{c} .
$$

We here have assumed that the coupling constants $\kappa=$ $3 /(8 \pi)\left(N \lambda_{i}^{2} \gamma_{a}\right)$ are equal for all transitions and that the two-photon detuning of all relevant Fourier components is small, such that $|\omega / \Omega| \ll 1 . \gamma_{a}$ is the common decay rate out of the upper levels, $\gamma_{0}$ is the decay rate of the coherence between the lower states, and $N$ is number density of atoms. Following the procedure of Ref. [13], we find for the non-vanishing noise correlations [15]

$$
\begin{aligned}
\left\langle f_{1}(z, \omega) f_{1}^{*}\left(z^{\prime}, \omega^{\prime}\right)\right\rangle & \simeq 4 \epsilon_{0}^{2} \frac{\kappa L}{c} \frac{\gamma_{0}}{\Omega^{2}} \delta\left(z-z^{\prime}\right) \delta\left(\omega+\omega^{\prime}\right), \\
\left\langle f_{1}(z, \omega) f_{2}\left(z^{\prime}, \omega^{\prime}\right)\right\rangle & \simeq 4 \epsilon_{0}^{2} \frac{\kappa L}{c} \frac{i}{\Delta} \delta\left(z-z^{\prime}\right) \delta\left(\omega+\omega^{\prime}\right), \\
\left\langle f_{2}(z, \omega) f_{2}^{*}\left(z^{\prime}, \omega^{\prime}\right)\right\rangle & \simeq 4 \epsilon_{0}^{2} \frac{\kappa L}{c} \frac{\gamma_{a}}{\Delta^{2}} \delta\left(z-z^{\prime}\right) \delta\left(\omega+\omega^{\prime}\right),
\end{aligned}
$$

where we have identified the quantization length with the length of the cell $L$. In the case described by Eq.(10) parametric oscillation occurs at $\omega=0$ when

$$
M(L, 0)=\cos (\eta L)+\left(\gamma_{0} \Delta\right) /\left(2|\Omega|^{2}\right) \sin (\eta L)=0,
$$

with $\eta=\kappa \sqrt{1 / \Delta^{2}-\gamma_{0}^{2} / 4|\Omega|^{4}}$. Hence oscillation can be achieved if $\Omega^{2}>\gamma_{0}|\Delta| / 2$. It should be noted that this is 
easily satisfied since $\gamma_{0}$ is the relaxation rate of a longlived ground-state coherence. Close to this oscillation condition the spectrum of the output field diverges 14

$$
n_{i}(\omega) \equiv \frac{c}{L} \int d \omega^{\prime} E_{i}^{*}(\omega) E_{i}\left(\omega^{\prime}\right) \sim \frac{1}{|M(L, \omega)|^{2}} .
$$

Note that in the limit $\gamma_{0} \rightarrow 0, \Delta \gg \gamma_{a}$ and $\omega \rightarrow 0$ the coefficients $a_{11}, a_{22}$, which correspond to the selfcoupling susceptibilities, become negligible and all noise correlations except for $\left\langle f_{1} f_{2}\right\rangle$ vanish. This corresponds to four-wave mixing with ideal noise properties [1,2]. The Stokes and anti-Stokes photons generated from vacuum $\left(E_{1}^{*}(0)=0, E_{2}(L)=0\right)$ possess in this case perfect quantum correlations, i.e. $100 \%$ squeezing. Hence the quantum fluctuations of a particular quadrature of the linear combination of output fields $\hat{d}_{\theta}(\omega) \equiv\left(\hat{E}_{1}(L, \omega)+\right.$ $\left.\hat{E}_{2}(0, \omega)\right) e^{i \theta} / \sqrt{2}$ can be almost completely suppressed near the threshold of parametric oscillation. We define the fluctuation spectrum of the combined mode at the output of the cell by

$$
S_{\theta}(\omega)=\frac{c}{4 L} \int d \omega^{\prime}\left\langle\left[\hat{d}_{\theta}(\omega)+\hat{d}_{\theta}^{\dagger}(\omega)\right],\left[\hat{d}_{\theta}\left(\omega^{\prime}\right)+\hat{d}_{\theta}^{\dagger}\left(\omega^{\prime}\right)\right]\right\rangle
$$

where $\langle a, b\rangle=\langle a b\rangle-\langle a\rangle\langle b\rangle$. As can be verified from the commutation relation $\left[\hat{E}_{1,2}(z, \omega), \hat{E}_{1,2}^{\dagger}\left(z, \omega^{\prime}\right)\right]=$ $(L / c) \delta\left(\omega-\omega^{\prime}\right)$ (which holds for Fourier-frequencies small compard to the carrier frequencies), the normalization is such that $S_{\theta}=1 / 4$ corresponds to the standard quantum limit. Using Eqs.(7) for the evaluation of normally ordered averages, and assuming that the system is close to the threshold $\left(|M|^{2}=|M(L, 0)|^{2} \ll 1\right)$ we find for the optimum phase $\theta=\pi / 4$

$$
S_{+}(0) \equiv S_{\pi / 4}(0)=\frac{|M|^{2}}{4}+\frac{\pi}{4}\left(2 \frac{\gamma_{0} \Delta}{|\Omega|^{2}}+\frac{\gamma_{a}}{\Delta}\right)
$$

where we have neglected by all but linear terms in $\gamma_{0} / \Omega^{2}$ and $\gamma_{a} / \Delta^{2}$. The first term on the rhs of the above expression is the residual quantum noise supressed by to nonlinear wave-mixing. The second term is an atomic noise contribution, which results from the finite relaxation rate of the ground state coherence and the associated absorption losses. Finally, the third contribution is the corresponding noise contribution due to the absorption of the far-detuned driving field. Choosing the optimum value for the detuning $\left(\Delta_{o p t}^{2}=\gamma_{a}|\Omega|^{2} /\left(2 \gamma_{0}\right)\right)$ we find that the maximum noise suppression is reached already before the oscillation threshold (for $|M|^{2}<\sqrt{\gamma_{0} \Delta / \Omega^{2}}$ ), and is given by:

$$
S_{+}(0) \rightarrow \pi\left(\frac{\gamma_{0} \gamma_{a}}{2|\Omega|^{2}}\right)^{1 / 2}
$$

The extent to which the parametric oscillator can be considered as ideal is determined by the absorption losses of the medium. In contrast to the usual two-level type systems [3] this absorption is here determined by the decay of the ground state coherence and by the detuning of one of the driving fields from single photon resonance $(\Delta)$. For

$$
|\Omega|^{2} \gg \gamma_{0} \gamma_{a}, \quad \Delta \gg \gamma_{a}, \Omega
$$

ideal correlations of Stokes and anti-Stokes fields are obtained.

For non-zero Fourier components the noise reduction deteriorates. It is clear that the bandwidth of squeezing is always on the order of the spectral width of the generated field, which becomes small near the oscillation threshold. For sufficiently small $\gamma_{0}, \omega$ and $|M|$ we can approximate $S(\omega)$ as:

$$
\begin{array}{r}
S_{\mathrm{opt}}(\omega) \approx \frac{\left(|M|^{2} / 2+\sqrt{1+\omega^{2} / \delta \omega_{0}^{2}}-1\right)^{2}}{|M|^{2}+\omega^{2} / \delta \omega_{0}^{2}} \\
+\frac{\pi}{4}\left(2 \frac{\gamma_{0} \Delta}{|\Omega|^{2}}+\frac{\gamma_{a}}{\Delta}\right) Z(\omega),
\end{array}
$$

where $\delta \omega_{0}=\Omega^{2} / \Delta$ and we have set $\theta$ to the optimum value for each Fourier frequency $\theta=\pi / 4-\kappa L \omega /\left(2 \Omega^{2}\right)$. $Z(\omega) \sim 1$ is some function, which is on the order of unity for arbitrary $\omega$. Its exact form is of no importance here. It follows directly from the above equation that squeezing is present for $\omega<\Omega^{2} / \Delta$, whereas maximum correlations, given by Eq. (18), occur within the bandwidth on the order of $\gamma_{0}\left(\Omega^{2} / \gamma_{a} \gamma_{0}\right)^{1 / 4}$. It is worth noting that in the present system all relevant spectral widths are determined by the atomic dispersion 10.

It is important to emphasize that the strong coupling regime corresponding to the conditions of ideal photon correlations or squeezing (19) can easily be realized even for very low driving-field intensities, since the groundstate relaxation rate $\gamma_{0}$ can be very small. For example, in the experiments involving hyperfine sublevels of the ground state of alkali vapors such as [7], this rate could be made as small as $1-100 \mathrm{~Hz}$. Even if a detuning $\Delta$ on the order of few tens of $\mathrm{MHz}$ is chosen, the Rabi frequencies corresponding to the value $\Omega^{2}=\gamma_{0}|\Delta|$ may well be close to the tens of $\mathrm{kHz}$ level. Under experimental conditions where the driving beams are in single spatial modes and diffraction limited, $\lambda \sim 1 \mu \mathrm{m}$, and where the optical pulse length is on the order of coherence life time $\left(\sim 1 / \gamma_{0}\right)$, the required Rabi frequencies correspond to only a few driving photons. In this limit efficient parametric interactions and mixing involving only few interacting light quanta may take place. This opens up a rather unique regime of nonlinear optics which allows, at least in principle, for single photon quantum control [10,11, and for "inelastic collisions" of single light quanta yielding correlated photons at different frequencies or polarizations. Furthermore, in such a regime the quantum nature of the driving fields as well as finite-size effects may become important [16]. 
In conclusion, we have demonstrated that resonant nonlinear interactions involving atomic coherence can be used for efficient generation of quantum-correlated electromagnetic fields with $100 \%$ squeezing without the use of cavities. We have shown that under appropriate conditions the resonant wave mixing process based on double$\Lambda$ atomic media can be regarded as ideal even for extremely low driving input powers. We expect these features of coherent atomic systems to be of interest in many areas of optics, spectroscopy and quantum control.

The authors gratefully acknowledge useful discussions with L. Hollberg, S. Harris, P. Hemmer, V. Sautenkov, and A. Zibrov and the support from the Office of Naval Research, the National Science Foundation, the Welch Foundation, the Texas Advanced Research and Technology Program and the Air Force Research Laboratories.

[1] D. F. Walls, Nature (London) 306, 141 (1983), D. F. Walls and G. J. Milburn, Quantum Optics, Springer, Belrin (1995); M. O. Scully and M. S. Zubairy, Quantum Optics, Cambridge University Press, Cambridge (1997).

[2] H. P. Yuen, and J. H. Shapiro; Opt. Lett. 4, 334 (1979); B. Yurke Phys. Rev. A 32, 300 (1985);

[3] R.E.Slusher, L.W.Hollberg, B.Yurke, J.C.Mertz, and J.F.Valley, Phys.Rev.Lett. 55, 2409 (1985); R. M. Shelby, M. D. Levenson, S. H. Perlmutter, R. G. DeVoe, and D. F. Walls, Phys.Rev.Lett. 57, 691 (1986); L. Wu, H. J. Kimble, J. L. Hall, and H. Wu, Phys.Rev.Lett. 57, 2520 (1986).

[4] K. Hakuta, L. Marmet, and B. P. Stoicheff, Phys. Rev. Lett. 66, 596 (1991); S.E. Harris, J.E. Field, and A. Imamoglu, Phys. Rev. Lett. 64, 1107 (1990); A. Kasapi, M. Jain, G.Y. Yin, and S.E. Harris, Phys. Rev. Lett. 74, 2447 (1995); M. Jain et al., Phys. Rev. Lett. 77, 4326 (1996); for review see S. E. Harris, Physics Today 50, \# 7, 36 (1997).

[5] P. R. Hemmer, D.P. Katz, J. Donoghue, M. CroninGolomb, M.S. Shahriar and P. Kumar, Opt. Lett. 20, 982 (1995).

[6] M. D. Lukin, P. Hemmer, M. Loeffler, and M. O. Scully, Phys. Rev. Lett. 81, 2675 (1998).

[7] A. S. Zibrov, M. D. Lukin, L. Hollberg, M. O, Scully, and V.L.Velichansky, "Parametric self-oscillation via resonantly enhanced multi-wave mixing", (in preparation).

[8] M.D. Lukin, M. Fleischhauer, M.O. Scully, and V.L. Velichansky, Opt. Lett. 23, 295 (1998).

[9] A. Imamoğlu, H. Schmidt, G. Woods, and M. Deutsch, Phys.Rev.Lett. 79, 1467 (1997).

[10] S. Harris and Y. Yamamoto, Phys.Rev.Lett. 81, 3611 (1998).

[11] M. Dunstan, S. Rebic, S. Tan, S. Parkins, M. Collett, and D. Walls, in Proceedings: Quantum Communication, Computing, and Measurement 2. (ed. by P. Kumar, G.M.
D'Ariano, and O. Hirota).

[12] For applications of squeezed states in quantum teleportation see S. L. Braunstein and H. J. Kimble, Phys.Rev.Lett. 80, 869 (1998).

[13] M. Fleischhauer and Th. Richter, Phys. Rev. A 51, 2430, (1995).

[14] Clearly at the threshold the linear approximation used in Eqs.(5) breaks down and higher order corrections need to be taken into account.

[15] Note that the $\delta$-function in space is due to vanishing corrleations between different atoms. The $\delta\left(\omega+\omega^{\prime}\right)$-term comes from double Fourier transforming $\delta\left(t-t^{\prime}\right)$ in $t$ and $t^{\prime}$.

[16] For these reasons direct extrapolation of the present results into this domain is strictly speaking inconsistent.

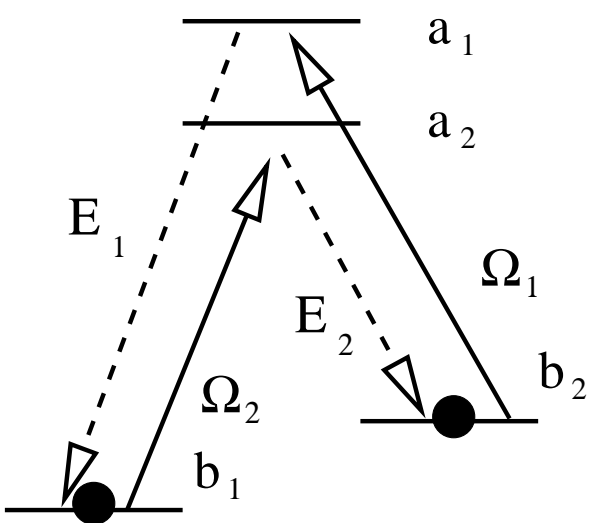

FIG. 1. Atoms in double $\Lambda$ configuration interacting with two classical driving fields $\left(\Omega_{1,2}\right)$ and two quantum fields $\left(E_{1,2}\right)$. All optical transitions are assumed to be radiatively broadened. 